\title{
Fine mapping of the sex locus in Salix triandra confirms a consistent sex determination mechanism in genus Salix
}

\author{
Wei $\mathrm{Li}^{1}$, Huaitong $\mathrm{Wu}^{1}$, Xiaoping $\mathrm{Li}^{1}$, Yingnan Chen ${ }^{1}$ and Tongming Yin ${ }^{1}$
}

\begin{abstract}
Salix triandra belongs to section Amygdalinae in genus Salix, which is in a different section from the willow species in which sex determination has been well studied. Studying sex determination in distantly related willow species will help to clarify whether the sexes of different willows arise through a common sex determination system. For this purpose, we generated an intraspecific full-sib $F_{1}$ population for $S$. triandra and constructed high-density genetic linkage maps for the crossing parents using restriction site-associated DNA sequencing and following a two-way pseudo-testcross strategy. With the established maps, the sex locus was positioned in linkage group XV only in the maternal map, and no sex linkage was detected in the paternal map. Consistent with previous findings in other willow species, our study showed that chromosome XV was the incipient sex chromosome and that females were the heterogametic sex in S. triandra. Therefore, sex in this willow species is also determined through a ZW sex determination system. We further performed fine mapping in the vicinity of the sex locus with SSR markers. By comparing the physical and genetic distances for the target interval encompassing the sex determination gene confined by SSRs, severe recombination repression was revealed in the sex determination region in the female map. The recombination rate in the confined interval encompassing the sex locus was approximately eight-fold lower than the genome-wide average. This study provides critical information relevant to sex determination in S. triandra.
\end{abstract}

\section{Introduction}

Flowering plants have evolved a considerably more complex sex determination system than animals, which have distinct germlines. Sexual diversity can first be observed in the various floral forms, ranging from hermaphroditic to monoecious and dioecious or even trioecious ${ }^{1}$. Approximately $5-10 \%$ of angiosperms are dioecious, with either heteromorphic or homomorphic sex chromosomes ${ }^{2}$. All sex chromosomes in dioecious plants harbor a sex determination region (SDR), which is characterized by suppressed recombination, leading to haplotype divergence ${ }^{3}$. Pseudo-autosomal regions (PARs)

\footnotetext{
Correspondence: Yingnan Chen (chenyingnan@njfu.edu.cn)

${ }^{1}$ The Key Lab of Cultivar Innovation and Germplasm Improvement of

Salicaceae, College of Forestry, Nanjing Forestry University, Nanjing 210037

China

These authors contributed equally: Wei Li, Huaitong Wu
}

are also present in plant sex chromosomes, with the physical lengths of PARs varying among different plants ${ }^{4}$. Different sex determination systems, including male heterogamety (XY system) and female heterogamety (ZW system), have been described in dioecious plants ${ }^{5}$. The overall interactions among elements such as plant hormones, genetic factors, and epigenetic modifications determine plant $\operatorname{sex}^{6}$.

Populus and Salix are sister genera in the Salicaceae family. These two lineages diverged at least 45 million years $\operatorname{ago}^{7,8}$. The chromosome number of willows and poplars is the same, and they do not exhibit a morphologically differentiated sex chromosome ${ }^{9,10}$. The Salicaceae family, whose young sex chromosomes have evolved from different autosomes, provides a valuable comparative system for studying sex differentiation in plants ${ }^{11}$. Multiple studies have reported that chromosome XIX is

\section{(c) The Author(s) 2020}

(c) (i) Open Access This article is licensed under a Creative Commons Attribution 4.0 International License, which permits use, sharing, adaptation, distribution and reproduction cc) in any medium or format, as long as you give appropriate credit to the original author(s) and the source, provide a link to the Creative Commons license, and indicate if changes were made. The images or other third party material in this article are included in the article's Creative Commons license, unless indicated otherwise in a credit line to the material. If material is not included in the article's Creative Commons license and your intended use is not permitted by statutory regulation or exceeds the permitted use, you will need to obtain permission directly from the copyright holder. To view a copy of this license, visit http://creativecommons.org/licenses/by/4.0/. 
the incipient sex chromosome in poplars ${ }^{12}$, and both XX/ $\mathrm{XY}^{13,14}$ and $\mathrm{ZW} / \mathrm{ZZ}^{12,15}$ sex determination systems have been observed in different poplar species. In poplars, the sex-determining locus has been mapped to either the peritelomeric region ${ }^{12}$ or the centromeric region of chromosome $\mathrm{XIX}^{14,16}$. However, no sex-determining genes have been identified among the Populus species analyzed so far, and only candidate genes have been reported. For example, the male-specific TOZ19 gene was identified as a sex determination candidate in P. tremula and $P$. tremuloides ${ }^{17}$. The sex determination region in poplar was found to contain a large number of $R$ genes $^{12,18}$, and thus, it was hypothesized that the emergence of the sex determination region might have been due to selective pressure arising from sex-specific floral pathogens $^{19}$. In P. balsamifera, the $P b R R 9$ gene exhibits male-biased methylation, indicating a role of epigenetic regulation in poplar sex determination ${ }^{20}$.

In willows, the sex determination locus has been consistently mapped to chromosome $\mathrm{XV}$, and only the $\mathrm{ZW}$ sex determination system has been observed ${ }^{21-24}$. The reconstruction of alternate haplotypes in the SDR revealed sequence divergence between the $\mathrm{Z}$ and $\mathrm{W}$ chromatids $^{22}$, and no homologous genes in the SDR have been found between the willow and poplar ${ }^{22,23}$. Pucholt et al. ${ }^{23}$ localized the sex determination locus to a $2.5-\mathrm{Mb}$ genomic region in S. viminalis that harbors 48 proteincoding genes. Further study showed that the SDR in S. viminalis is of limited size $(\sim 804 \mathrm{~kb})$ and exhibits a higher SNP density in females ${ }^{25}$. Pseudogenization and the accumulation of repetitive elements in the SDR suggest that the fundamental process of sex chromosome formation occurred very swiftly after recombination ceased $^{11}$. In a recent study, the SDR of S. purpurea was found to contain large palindromic repeats, and the SpRR9 gene was considered a putative candidate for controlling sex determination through the modulation of the cytokinin signaling pathway ${ }^{26}$. Whether willow exhibits a relatively conserved sex determination system needs to be explored in more willow species.

S. triandra is a shrub willow belonging to section Amygdalinae in genus Salix. It is distributed widely from Japan to western Europe ${ }^{27}$. More recently, S. triandra has received attention because of its potential implications in insect resistance ${ }^{28,29}$. Due to the reproductive efficiency, easy cultivation, and small genome, $S$. triandra is suitable for obtaining additional information to better understand sex determination in dioecious plants. In this study, S. triandra is used to provide new evidence of the sex determination mechanism in willow. Our purpose is to clarify whether the previously reported willow sex determination system also functions in a willow species belonging to a different section of genus Salix.

\section{Materials and methods}

Plant materials and DNA extraction

The mapping population, which consisted of 152 fullsib $F_{1}$ progenies, was established in 2013 by crossing the S. triandra female clone "DB447" with the male clone "DB134". "DB447" and "DB134" were sampled from the Maoer Mountain in Heilongjiang Province of China (permissions were granted by the local administration). The parental clones and progeny were maintained at the Baima Forest Farm in Lishui in Jiangsu Province, China. Genomic DNA was extracted from the young leaves of each individual by using an E.Z.N.A. Plant DNA Kit (Omega Bio-tek, Norcross, GA, USA). DNA quality was assessed by $1 \%$ agarose gel electrophoresis, and the DNA concentration was measured with a Nanodrop 2000 (Thermo Scientific, MA, USA).

\section{Library construction and sequencing}

The whole-genome sequencing (WGS) was conducted with the two crossing parents, and restriction siteassociated DNA (RAD) sequencing was performed for 152 progenies of the mapping population. For the crossing parents, two paired-end libraries with $300-500 \mathrm{bp}$ insert sizes were constructed separately according to the standard protocol of Illumina (Illumina). For each progeny, the RAD library was prepared following the method described by Baird et al. ${ }^{30}$ with minor modifications. Briefly, $300 \mathrm{ng}$ of genomic DNA from each progeny was digested separately by using $5 \mathrm{U}$ of Tap I (Takara Bio, Japan) at $37^{\circ} \mathrm{C}$ for $60 \mathrm{~min}$, and then the P1 adapter, which contained a forward primer site, an Illumina sequencing primer site and a barcode ( $4-8 \mathrm{bp}$ ), was ligated to the fragments. Subsequently, the P1-ligated fragments of all samples $(1 \mu \mathrm{L}$ each) were pooled and then randomly sheared (Bioruptor) to an average size of $500 \mathrm{bp}$.

The entire sample was separated using $1 \%$ agarose gel electrophoresis, and the DNA fraction corresponding to 300-700 bp was isolated using an E.Z.N.A. Gel Extraction Kit (Omega Bio-tek, USA). The purified fragments were subjected to end repair and the $3^{\prime}$-end addition of dATP overhangs, followed by the ligation of a P2 adapter containing a reverse primer site and an Illumina sequencing primer site. Finally, the RAD library was selectively enriched by PCR amplification with the P1-forward primer and P2reverse primer, and the 300-700 bp amplicons were purified again with the Gel Extraction Kit (Omega Bio-tech, USA).

Both WGS and RAD sequencing were performed on the Illumina HiSeq $\mathrm{X}$ Ten platform (Illumina, USA) at Shanghai Major Biological Medicine Technology following the manufacturer's protocol (Illumina).

\section{Sequence analysis and nucleotide variant identification}

Raw reads were assigned to each individual based on the unique barcodes and then subjected to quality control, 
adapter trimming and read filtering by using FASTP (version 0.6.0, https://github.com/OpenGene/fastp). Reads that contained $>40 \%$ low-quality bases (base quality value $<15)$ or $>10 \% \mathrm{~N}$ bases were discarded. Sequences shorter than $30 \mathrm{bp}$ after trimming were also removed.

The resulting high-quality reads were mapped to the reference genome of S. purpurea v1.0 (DOE-JGI, http:// phytozome.jgi.doe.gov/pz/portal.html\#!info?alias= Org_Spurpurea) by using BWA (version 0.7.16, http://bio-bwa. sourceforge.net/) software ${ }^{31}$ with the default parameters. GATK Haplotype Caller ${ }^{32}$ was used to call nucleotide variants, including SNPs and InDels, which were further filtered according to GATK Best Practices ${ }^{33}$.

\section{Linkage map construction}

To obtain high-quality linkage maps, all the filtered genetic markers were further screened based on the following criteria: (1) average sequence depth $>5 \times$ in the parents and $>4 \times$ in the progeny; (2) heterozygous as least in one parent; (3) present in $\geq 70 \%$ progeny; and (4) following a Mendelian segregation ratio. Markers with significant segregation distortion $\left(X^{2}\right.$ test, $\left.P<0.05\right)$ were excluded from linkage map construction.

The integrated linkage analysis was performed by using JoinMap 5.0 (https://www.kyazma.nl/index.php/JoinMap/ ), and a logarithm of odds (LOD) score threshold of 4.0 was employed to establish linkage groups (LGs). The female and male maps were constructed with a two-way pseudo-testcross strategy. The LGs were nominated according to the alignment of the mapped markers with the S. purpurea v1.0. genome assembly. The genetic distance between markers was estimated using the Kosambi mapping function ${ }^{34}$. The marker distribution in each LG was analyzed using the sliding window $(10 \mathrm{cM})$ approach $^{35}$. The quality of the genetic map was assessed using a haplotype map and a heat map ${ }^{36}$.

\section{Linkage analysis of the sex locus}

The sex of the plants was visually recorded for the 152 progenies. Among these progenies, 77 were female and 75 were male. The phenotypic data were included in the data matrix of each parent and scored as a testcross marker. Based on the established genetic maps, the sex locus was mapped as a segregating morphological marker with MapMaker software (version 3.0). To verify the accuracy of the positioning interval, we designed SSR markers with a physical distance of $4 \mathrm{Mb}$ upstream and downstream from two SNP markers that were completely linked with sex.

\section{Results \\ DNA sequencing data}

For each parent, WGS yielded $9.65 \mathrm{~Gb}$ of clean sequencing data on average, and the sequencing depth was $\sim 20 \times$ genome coverage (Fig. 1). After quality control, a total of $136.96 \mathrm{M}$ clean reads were obtained from the two parents, with an average Q30 ratio of $90.03 \%$ and an average guanine-cytosine (GC) content of $37.25 \%$ (Table 1). For the $152 \mathrm{~F}_{1}$ offspring, a total of $504.21 \mathrm{~Gb}$ of clean data were generated, including $3622.24 \mathrm{M}$ of high-quality clean reads with a length of $150 \mathrm{bp}$ (Table 1). The number of the clean reads ranged from 11.03 to $53.06 \mathrm{M}$ among different offspring, with an average of $23.83 \mathrm{M}$. The average sequencing depth for each progeny was approximately $7.53 \times$, varying from $4.06 \times$ to $16.78 \times$ (Fig. 1 ). The average Q30 ratio was $88.88 \%$, and the GC content was $41.58 \%$ (Table 1).

\section{Nucleotide variant discovery and genotyping}

The high-quality reads obtained from all samples were separately mapped to the reference genome of $S$. purpurea v1.0, and the mapped ratio ranged from $46.89 \%$ to $88.04 \%$, with an average of $80.34 \%$ (Supplementary Table S1). All mapped reads were used for SNP calling, and a total of $1,150,885$ putative nucleotide variant loci were detected in both parents. Based on genotyping information and the stringent filtering criteria described in the "Materials and methods' section, 22,830 high-quality markers were retained from the whole $F_{1}$ population, including 20,695 SNPs and 2135 InDels (Table 2). Among these markers, 9188 (8301 SNPs and 887 InDels) were only maternally informative (nn×np), 9089 (8297 SNPs and 792 InDels)

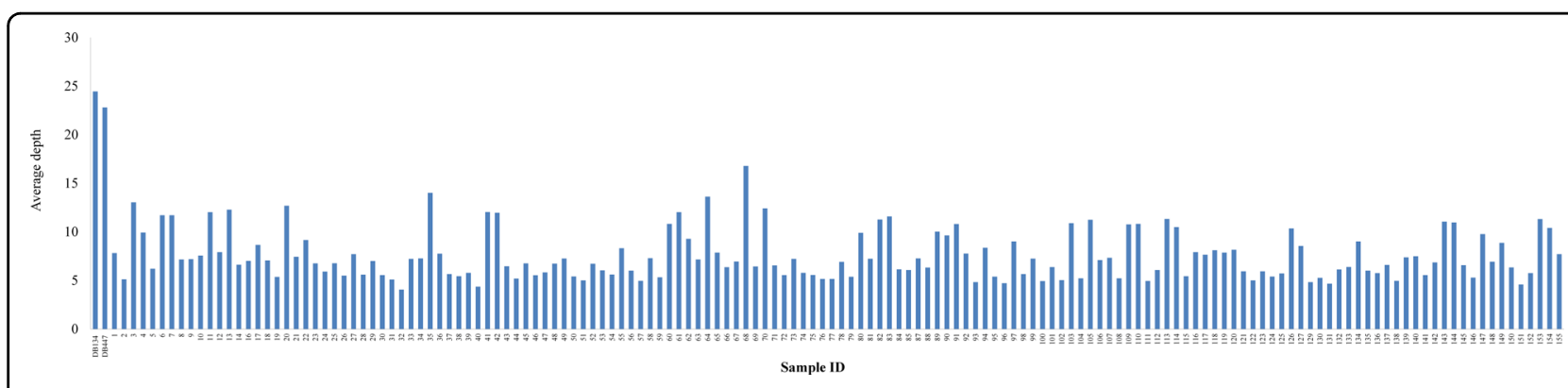

Fig. 1 Sequencing depth of each sample. The sampled accessions are indicated on the $x$-axis 
Table 1 Statistics of the WGS and RAD sequencing results in S. triandra

\begin{tabular}{llccccc}
\hline Sample & Raw data $(\mathrm{Gb})$ & Clean data $(\mathrm{Gb})$ & Clean reads (M) & Clean data percentage (\%) & Q30 (\%) & GC (\%) \\
\hline DB134 & 10.94 & 9.90 & 70.07 & 90.49 & 90.43 & 36.40 \\
DB447 & 10.49 & 9.41 & 66.89 & 89.70 & 89.63 & 38.10 \\
Progeny & 603.69 & 504.21 & 3622.24 & 83.52 & 88.88 & 41.58 \\
Total & 625.12 & 523.52 & 3759.20 & - & - \\
\hline
\end{tabular}

Table 2 Summary of the marker types and numbers of markers used for genetic map construction

\begin{tabular}{lll}
\hline Marker type & SNP number & InDel number \\
\hline efxeg $(1: 1: 1: 1)$ & 6 & 2 \\
hkxhk $(1: 2: 1)$ & 4091 & 454 \\
Im×II (1:1) & 8297 & 792 \\
nnxnp (1:1) & 8301 & 887 \\
Total & 20,695 & 2135 \\
\hline
\end{tabular}

Note: The 'Im $\times I I$ ' and ' $\mathrm{n} \times \mathrm{np}$ ' segregation types represent markers that are heterozygous only in the paternal or maternal parent, respectively.

were only paternally informative $(\operatorname{lm} \times 1 \mathrm{ll})$, and the remaining 4553 (4097 SNPs and 456 InDels) were intercrossing markers in both parents (Table 2).

\section{Construction and evaluation of the high-density linkage map}

We constructed the paternal, maternal and consensus maps for S. triandra separately. The maternal map was $2193.78 \mathrm{cM}$ in length, with LG sizes ranging from $91.25 \mathrm{cM}$ (LG13) to $141.64 \mathrm{cM}$ (LG14) (Table 3). The paternal map was $2381.93 \mathrm{cM}$ in length, with LG sizes varying from $90.34 \mathrm{cM}$ (LG17) to $148.70 \mathrm{cM}$ (LG1) (Table 3).

All 22,830 markers that segregated as ef $\times$ eg, hk $\times$ hk, $\operatorname{lm} \times$ ll or $n n \times n p$ were used to generate a consensus map for $S$. triandra. At the LOD threshold of 4.0, all of these markers were successfully grouped into 19 LGs (Fig. 2 and Fig. S1). The number of LGs was consistent with the haploid chromosome number of willows $(2 n=38)$. The established consensus map covered a genetic distance of $2239.71 \mathrm{cM}$, with LG sizes varying from $96.87 \mathrm{cM}$ (LG6) to $145.29 \mathrm{cM}$ (LG14) (Table 3). The marker distribution along each LG was evaluated by counting the number of marker bins and all mapped markers using a sliding window of $10 \mathrm{cM}$. The average number of marker bins ranged from 18.00 to 69.87 , with the average number of mapped markers varying from 43.93 to 154.27 . The window with the highest density (26.7 markers per cM) was found in LG16 (Fig. 3).
Haplotype maps, which revealed the missing data and recombination events of each individual intuitively, were generated for each LG in the 152 offspring (Supplementary Fig. S2). The percentages of missing data and double crossovers were less than $0.30 \%$ and $0.23 \%$, respectively. Based on the pairwise recombination values of the markers grouped in each LG, heat maps were generated for the 19 LGs (Supplementary Fig. S3). All the heat maps demonstrated a clear trend in which the pairwise linkage generally decreased with an increase in genetic distance between the mapped markers, indicating that the markers in each LG were precisely mapped and ordered.

\section{Collinearity between the genetic map and reference genome}

All the mapped markers were aligned to the S. purpurea v1.0 genome to estimate the physical distances of the markers and to assess the collinearity between the genetic map and reference genome. In general, high collinearity was observed between the markers and the corresponding chromosomes (Fig. 4), with the Spearman rank correlation coefficient ranging from 0.99-1.00. However, there were also LGs showing discrepancies in some narrow regions (e.g., LGs 14, 18 and 19), which might have been due to different recombination rates, missing data or compromised marker orders in the consensus map.

The mapped markers in the integrated genetic map covered at least $96.95 \%$ of the physical length of the reference genome (Table 4). The genetic-to-physical distance ratios ranged from $3.78 \mathrm{cM} / \mathrm{Mb}$ (LG6) to $11.65 \mathrm{cM} /$ Mb (LG14) (Table 4). The marker density along each chromosome ranged from 28.81 to 70.64 markers/Mb, averaging 49.18 markers/Mb (Table 4).

\section{Mapping the sex locus and gene content in the confined genetic interval}

The mapping results showed that the sex locus could only be mapped in the maternal map $27.07 \mathrm{cM}$ from the telemetric end of chromosome XV (Fig. 5a), and no linkage with sex was detected in the paternal map. Using the sequences of the SNP markers co-segregating with sex, we obtained genome sequences in the confined genetic interval and developed upstream and downstream 
Table 3 Summary of the linkage map of S. triandra

\begin{tabular}{|c|c|c|c|c|c|c|c|c|}
\hline \multicolumn{3}{|c|}{ Maternal map } & \multicolumn{3}{|c|}{ Paternal map } & \multicolumn{3}{|c|}{ Consensus map } \\
\hline Group & Total marker & Total distance (cM) & Group & Total marker & Total distance (cM) & Group & Total marker & Total distance (cM) \\
\hline FLG1 & 837 & 118.00 & MLG1 & 805 & 148.70 & LG1 & 1355 & 143.26 \\
\hline FLG2 & 788 & 132.74 & MLG2 & 882 & 124.02 & LG2 & 1402 & 142.75 \\
\hline FLG3 & 725 & 136.62 & MLG3 & 713 & 137.28 & LG3 & 1183 & 103.54 \\
\hline FLG4 & 481 & 101.33 & MLG4 & 549 & 136.98 & LG4 & 869 & 142.71 \\
\hline FLG5 & 782 & 128.83 & MLG5 & 793 & 104.40 & LG5 & 1296 & 110.57 \\
\hline FLG6 & 872 & 129.78 & MLG6 & 794 & 125.72 & LG6 & 1387 & 96.87 \\
\hline FLG7 & 602 & 138.12 & MLG7 & 618 & 140.52 & LG7 & 998 & 102.74 \\
\hline FLG8 & 729 & 113.96 & MLG8 & 667 & 136.27 & LG8 & 1164 & 140.66 \\
\hline FLG9 & 524 & 91.60 & MLG9 & 576 & 125.62 & LG9 & 912 & 98.54 \\
\hline FLG10 & 775 & 91.70 & MLG10 & 827 & 107.81 & LG10 & 1377 & 101.10 \\
\hline FLG11 & 800 & 129.95 & MLG11 & 827 & 128.47 & LG11 & 1353 & 116.95 \\
\hline FLG12 & 512 & 127.17 & MLG12 & 612 & 103.98 & LG12 & 967 & 97.31 \\
\hline FLG13 & 770 & 91.25 & MLG13 & 719 & 117.28 & LG13 & 1231 & 129.91 \\
\hline FLG14 & 491 & 141.64 & MLG14 & 527 & 123.03 & LG14 & 874 & 145.29 \\
\hline FLG15 & 810 & 105.68 & MLG15 & 713 & 133.58 & LG15 & 1269 & 125.36 \\
\hline FLG16 & 1401 & 110.86 & MLG16 & 1432 & 123.74 & LG16 & 2314 & 144.95 \\
\hline FLG17 & 702 & 105.81 & MLG17 & 725 & 90.34 & LG17 & 1170 & 139.85 \\
\hline FLG18 & 667 & 96.65 & MLG18 & 580 & 132.16 & LG18 & 1094 & 120.19 \\
\hline FLG19 & 374 & 102.09 & MLG19 & 382 & 142.02 & LG19 & 615 & 137.16 \\
\hline Total & 13,642 & 2193.78 & Total & 13,741 & 2381.93 & Total & 22,830 & 2239.71 \\
\hline
\end{tabular}

sex-linked simple sequence repeat (SSR) markers. In total, seven SSR markers co-segregating with the sex locus were generated in the SDR on chromosome XV of the female (Fig. 5c), and the confined interval encompassing the sex locus (IESL) was bounded by SSR markers wssr304 and wssr470, with spacing of $5.9 \mathrm{cM}$ (Fig. 5b). Based on the reference genome of $S$. purpurea, the confined IESL corresponded to a $6.5-\mathrm{Mb}$ genomic region on chromosome 15 . On average, a $1 \mathrm{cM}$ genetic length contains a $140 \mathrm{~kb}$ sequence in the willow genome. Thus, the recombination rate in the confined IESL of the female is approximately eight-fold lower than the genome-wide average.

The target region harbored 249 genes. Gene Ontology (GO) terms clarified that genes involved in metabolic processes, cellular processes, single organism processes, reproductive processes and biological organization were the most represented groups (Fig. 6). Six genes were associated with microtubule motor activity (GO:0003777), and six genes were associated with microtubule binding activity (GO:0008017). EVM0038350 has a methyltransferase domain that may be related to DNA methylation. Salix_newGene_2 is a homologous gene of LRR receptor-like serine/threonine-protein kinase ERL1 in Arabidopsis thaliana, which is important for anther lobe formation. The EVM0005130 gene contains a Myblike DNA-binding domain that plays an important role in regulating anther and pollen development; EVM0045351 contains a mitogen-activated protein kinase (MAP kinase) domain that may function as a regulator of pollen development and germination.

In addition to the genes involved in flower organ development, flower development-associated miR156 was also found in the confined IESL. miR156 has emerged as the most important regulator in the vegetative phase change and the vegetative-to-reproductive transition in both Arabidopsis and maize. The candidate genes related to flower development are listed in Table 5.

\section{Discussion}

The Salicaceae family is a valuable model system for revealing the origin and evolution of plant sex chromosomes. These genera are widely distributed around the 
a

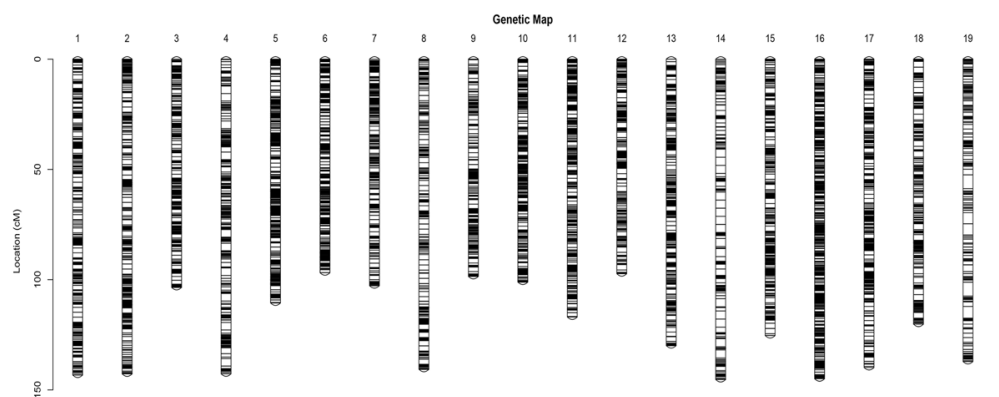

b

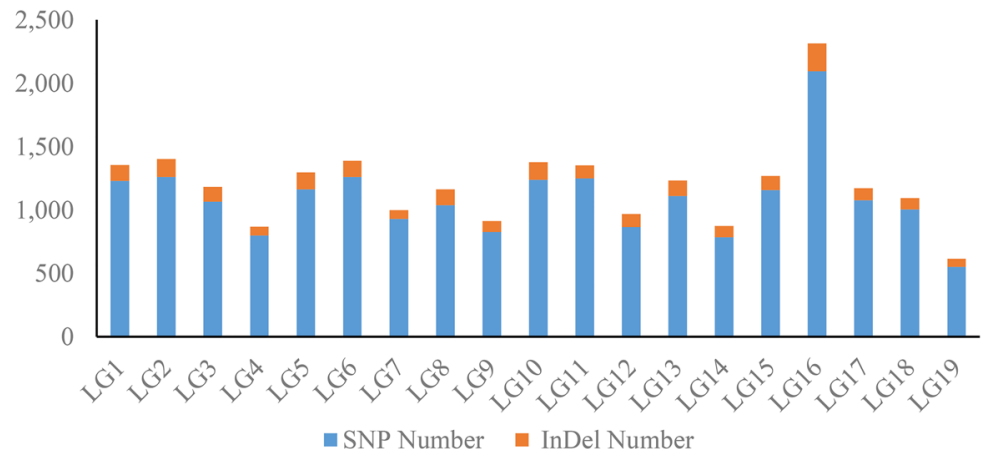

Fig. 2 The consensus genetic map of S. triandra. a Distribution of mapped markers within each linkage group of S. triandra. A black line indicates a SNP/InDel marker. $\mathbf{b}$ The number of SNPS and InDels in each linkage group
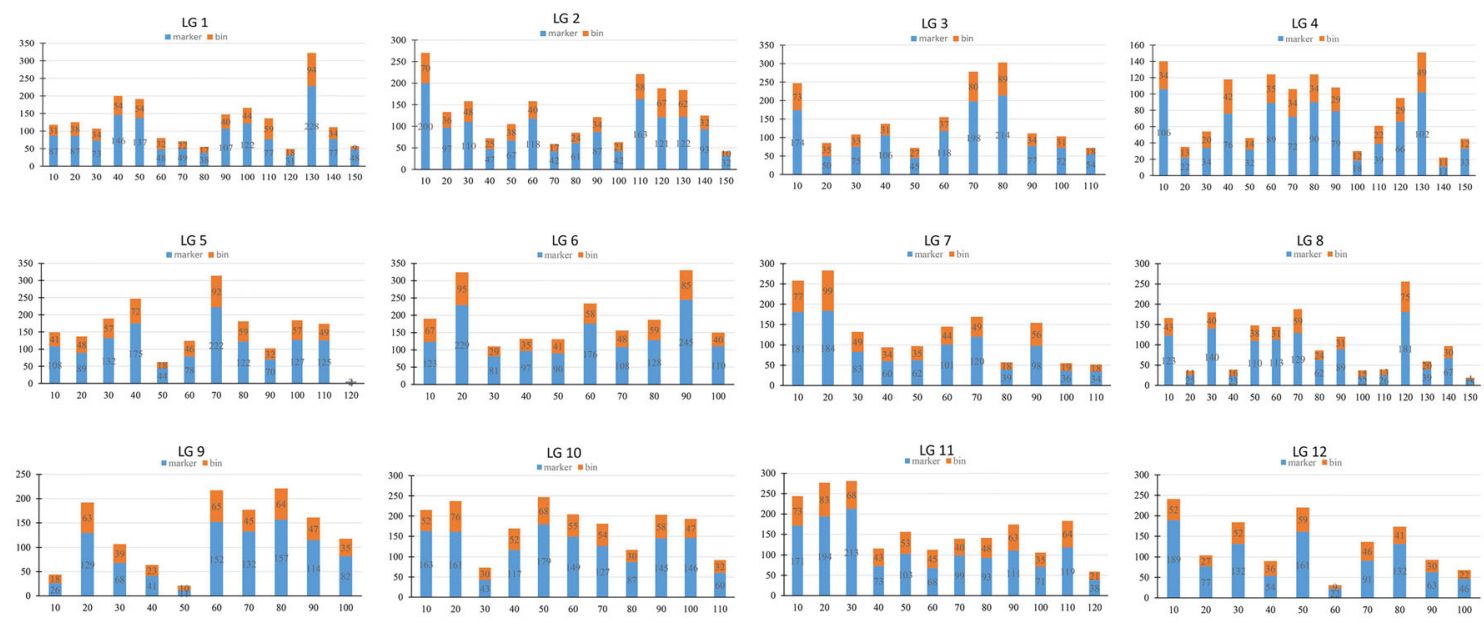

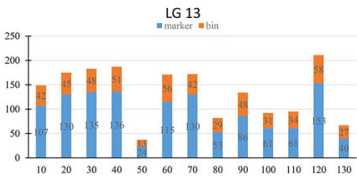
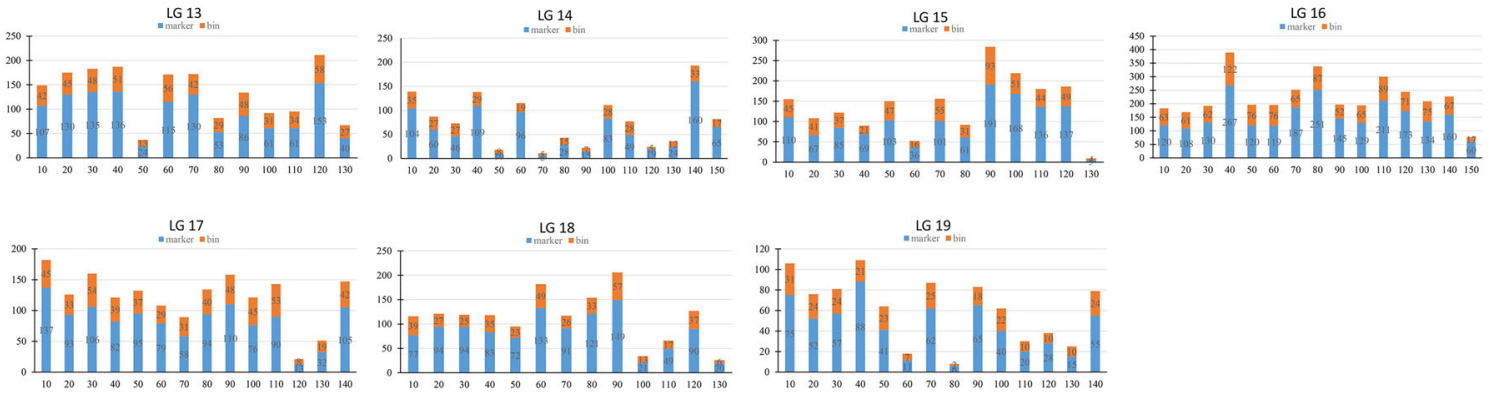

Fig. 3 Marker distribution analysis of the consensus map. The numbers of markers and marker bins are indicated with blue and yellow bars, respectively 


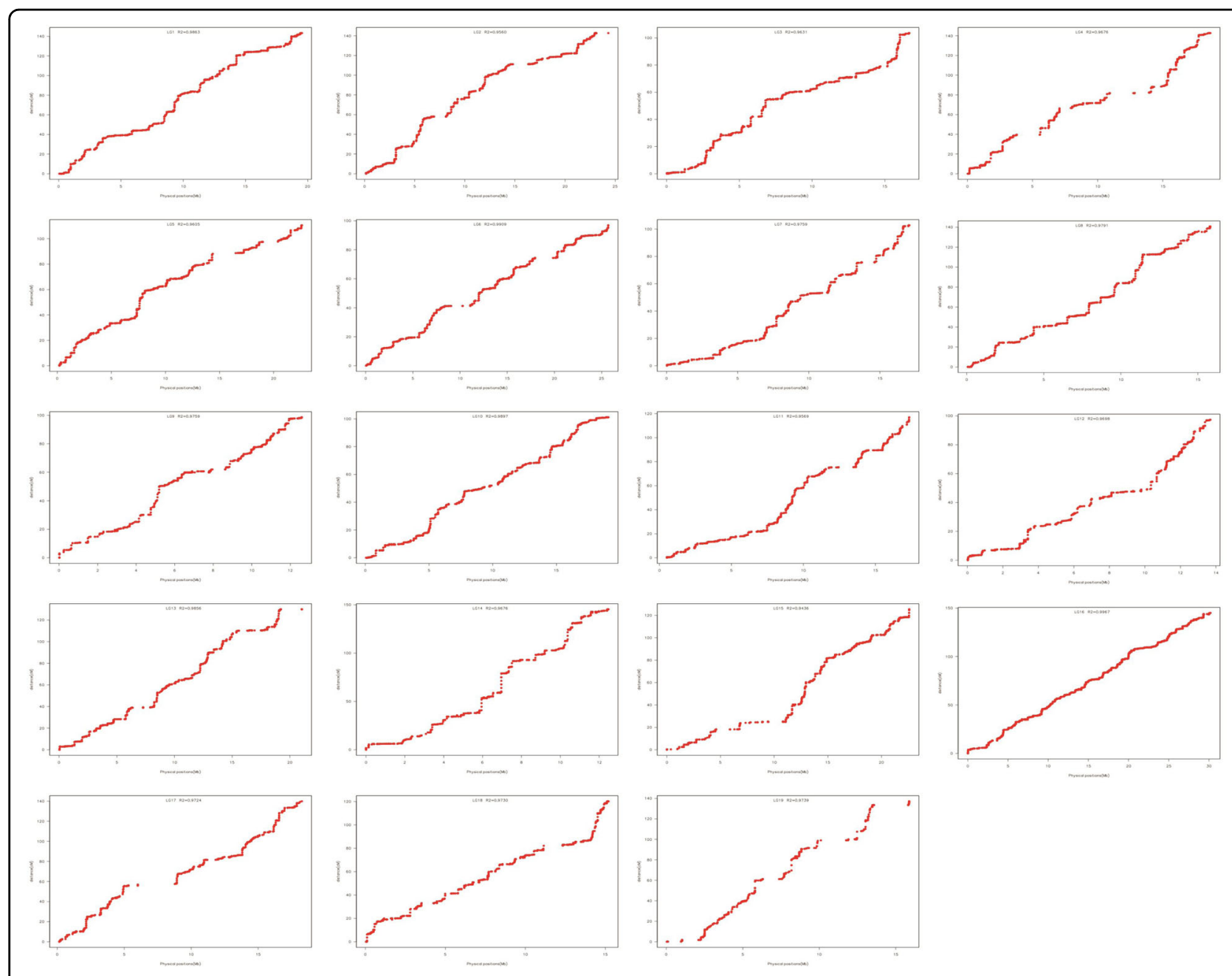

Fig. 4 Collinearity analysis between the integrated S. triandra genetic map and the S. purpurea v1.0. genome. The marker position in the genome is shown on the $x$-axis; the marker position in each linkage group is shown on the $y$-axis

globe, representing a diverse assemblage of subtrees, shrubs $^{37}$ and catkin-bearing trees. Nearly all species in genera Salix (Ca. 500 species) ${ }^{38}$ and Populus (Ca. 30 spe(ies) $)^{39}$ are dioecious, though obvious heteromorphic sex chromosomes have not yet evolved. Furthermore, the two lineages share a well-preserved whole-genome duplication ${ }^{7,8}$ and show an ongoing propensity toward polyploid formation $^{40,41}$, which facilitates the exploration of the relationship between polyploidy and sex chromosome evolution ${ }^{42}$.

In the past decade, there have been many reports on the sex determination mechanisms of poplars. In both $P$. deltoides and P. nigra, which are from section Aigeiros, the SDR is located at the proximal telomeric end of chromosome $\mathrm{XIX}^{12,13}$. In P. tremuloides, $P$. tremula and $P$. alba, all of which belong to section Populus, a pericentromeric region of chromosome $\mathrm{XIX}^{14,15,43}$ was determined to be the SDR. Both female heterogamety ${ }^{12,15}$ and male heterogamety have been reported ${ }^{13,43}$. Recently, sex determination was explored in $52 P$. trichocarpa (section Tacamahaca) and 34 P. balsamifera (section Populus) individuals by using the genome-wide association study. A total of 650 sex-associated SNPs were found to be heterozygous in males, indicating an XY sex determination system in these two species ${ }^{44}$. In genus Salix, the SDR is confined to the centromeric region of chromosome XV in S. viminalis (section Viminella) 22,23,45 $^{2}$ and $S$. suchowensis (section Helix) $)^{22,23,45}$. Female heterozygosis predominates in the SDRs of these species, suggesting a ZW sex determination system in Salix. Furthermore, no candidate genes in the willow SDR are orthologous to those in the poplar $\mathrm{SDR}^{22,23}$. Current research is not 
Table 4 Statistics of the collinearity analysis between the integrated S. triandra genetic map and the $S$. purpurea v1.0. genome

\begin{tabular}{|c|c|c|c|c|}
\hline LG & $\begin{array}{l}\text { Physical } \\
\text { coverage (\%) }\end{array}$ & $\begin{array}{l}\text { Genetic distance/ } \\
\text { physical distance } \\
\text { (cM/Mb) }\end{array}$ & $\begin{array}{l}\text { Density } \\
\text { (markers/ } \\
\mathrm{Mb} \text { ) }\end{array}$ & $\begin{array}{l}\text { Spearman } \\
\text { correlation }\end{array}$ \\
\hline 1 & 99.54 & 7.35 & 55.10 & 0.99 \\
\hline 2 & 99.34 & 5.91 & 65.73 & 1.00 \\
\hline 3 & 99.85 & 6.19 & 57.08 & 0.99 \\
\hline 4 & 99.72 & 7.67 & 42.89 & 0.99 \\
\hline 5 & 98.75 & 4.93 & 33.47 & 1.00 \\
\hline 6 & 99.63 & 3.78 & 28.81 & 1.00 \\
\hline 7 & 99.78 & 6.03 & 35.61 & 1.00 \\
\hline 8 & 98.98 & 8.94 & 53.54 & 0.99 \\
\hline 9 & 99.21 & 7.83 & 40.35 & 0.99 \\
\hline 10 & 99.62 & 5.32 & 46.90 & 1.00 \\
\hline 11 & 96.95 & 6.95 & 65.70 & 1.00 \\
\hline 12 & 99.38 & 7.12 & 51.26 & 0.99 \\
\hline 13 & 99.87 & 6.18 & 42.68 & 0.99 \\
\hline 14 & 99.75 & 11.65 & 60.42 & 0.99 \\
\hline 15 & 99.91 & 5.55 & 42.30 & 1.00 \\
\hline 16 & 99.96 & 4.81 & 70.64 & 1.00 \\
\hline 17 & 99.11 & 7.73 & 58.53 & 0.99 \\
\hline 18 & 99.62 & 7.93 & 52.38 & 0.99 \\
\hline 19 & 99.76 & 8.66 & 31.05 & 0.99 \\
\hline Mean & 99.41 & 6.87 & 49.18 & 0.99 \\
\hline
\end{tabular}

sufficient to demonstrate whether the sex determination mechanisms of the two lineages are related.

In this study, we sought to explore the SDR in an additional willow species, S. triandra (section Amygdalinae). In the $S$. triandra SDR, obvious recombination suppression was observed in the female. Recombination suppression, which means that homologous chromosomes cannot pair and undergo recombination, is an important component of sex chromosome evolution ${ }^{46}$. In both advanced and primitive sex chromosomes, the suppression of recombination in chromosomal regions is observed in numerous plant species. It has been reported that both the $\mathrm{X}$ and $\mathrm{Y}$ chromosomes of Actinidia chinensis var. chinensis exhibit a similar pattern of restricted recombination: approximately one-third of the sex chromosome (terminal $\sim 6 \mathrm{Mb}$ of chromosome $\mathrm{XXV}$ ) spans the SDR and shows severe recombination suppression, while the remaining section undergoes normal recombination ${ }^{47}$. Recombination suppression is also observed in the SDR in papaya, and the nonrecombining region of the $\mathrm{Y}$ chromatid differed greatly from the corresponding region of the $\mathrm{X}$ chromatid due to two large inversions ${ }^{48}$. In hop (Humulus lupulus), an approximately four-fold reduction in recombination is found on the $\mathrm{Y}$ chromosome compared with the $\mathrm{X}$ chromosome linkage map ${ }^{49}$. A study on Silene alba also found that almost the entire SDR of the sex chromosome showed heavily suppressed recombination ${ }^{50}$. Chromosomal inversion, heterochromatinization, and DNA methylation may be the underlying mechanisms of recombination suppression ${ }^{51}$. Large-scale heterochromatinization and loss-of-function regions are found in the $\mathrm{Y}$ chromosome of sorrel ${ }^{52}$. DNA methylation may defend against the insertion of DNA repeats derived from transposons and speed up heterochromatinization in specific regions of sex determination ${ }^{53}$. The methylation and heterochromatin levels of the male-specific DNA region of the $\mathrm{Y}$ chromosome are higher than those of the corresponding region of the $\mathrm{X}$ chromosome in Papaya $^{53}$. The reduced recombination in sex chromosomes leads to the differentiation of their structure and function; male- or female-specific sequences accumulate in chromosomes, leading to a high degree of degeneration in sex chromosomes ${ }^{51,54}$. After a long period of evolutionary accumulation, autosomes may ultimately evolve into morphologically and functionally different sex chromosomes. In this study, we performed fine local mapping with SSR markers designed with sequences from the confined IESL of $S$. triandra, and severe recombination repression in the SDR was observed between the two sexes. Similar features are observed in S. suchowensis $^{22}$, S. viminalis ${ }^{23}$ and S. purpurea ${ }^{55}$.

Dioecy has evolved hundreds of times from a hermaphroditic ancestor, and different genes may be involved in this process ${ }^{56,57}$, leading to great challenges in identifying the particular sex determination genes of different taxa of plants. The identification of sexdetermining genes is of great significance to reveal the mechanism underlying sex determination in flowering plants. Therefore, genes involved in floral development located in the SDR are good putative candidates. Akagi et al. ${ }^{58}$ found that the $S y G I$ gene, located in the Y-specific region, was involved in carpel development. In Diospyros lotus (XY system), the only identified sex-determining gene, OGI, which is located in a male-specific region, encodes 21-bp small RNAs targeting the autosomal gene $M e G I$, which acts as a maleness suppressor ${ }^{59}$.

In this study, a single SDR was physically located within a physical interval of $6.5 \mathrm{Mb}$ in chromosome $\mathrm{XV}$ in S. triandra, which shows clear female heterogamety. This 


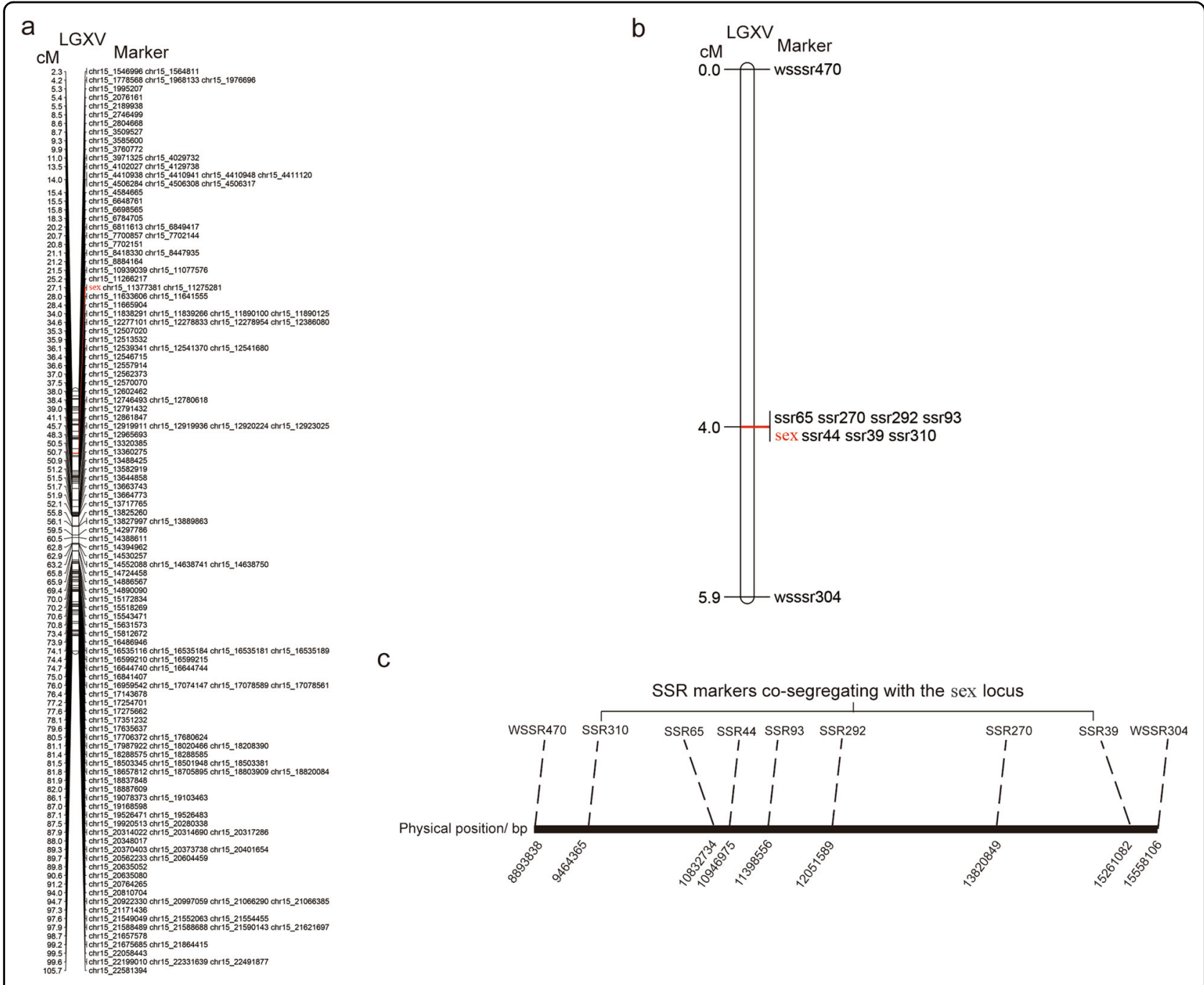

Fig. 5 Locating the sex locus of $\boldsymbol{S}$. triandra. a Linkage analysis mapping the sex locus of S. triandra within LGXV of the female map. $\mathbf{b}$ Fine positioning of the SSR markers in the vicinity of the sex locus in S. triandra. $\mathbf{c}$ The physical positions of SSR markers in the confined IESL

observation is consistent with findings in other willow species $^{22,23}$. However, the estimated size of the SDR should not be considered definitive, due to the limited resolution of the genetic maps. In the confined IESL of S. triandra, six genes exhibit the molecular function of microtubule motor activity, which is involved in male reproductive development and function ${ }^{60}$. Similar gene groups are found in the S. purpurea $\mathrm{SDR}^{55}$. Another gene, EVM0005130, which contains a Myb-like DNA-binding domain, deserves special attention because it plays an important role in regulating anther and pollen development ${ }^{61}$. We also detected the interesting gene EVM0045351, containing a mitogen-activated protein kinase (MAP kinase) domain, which has been proposed to function as a regulator of both pollen development and germination $^{62}$. Increasing numbers of studies have demonstrated that miRNAs play critical roles in regulating plant growth and stress responses as well as plant reproductive development. miRNA156 and 159, which are related to plant flower development, have been identified in the SDR.

In conclusion, the present study developed high-density linkage maps for $S$. triandra. The mapping of the sex locus revealed female heterogamety, indicating that sex in this willow species is determined through a $\mathrm{ZW}$ determination system. We confined the sex determination locus of $S$. triandra to a $6.5 \mathrm{Mb}$ genomic region that harbors 249 genes and 22 miRNAs. The region contains several promising sex determination candidates, which are worthy of special attention in future studies. 


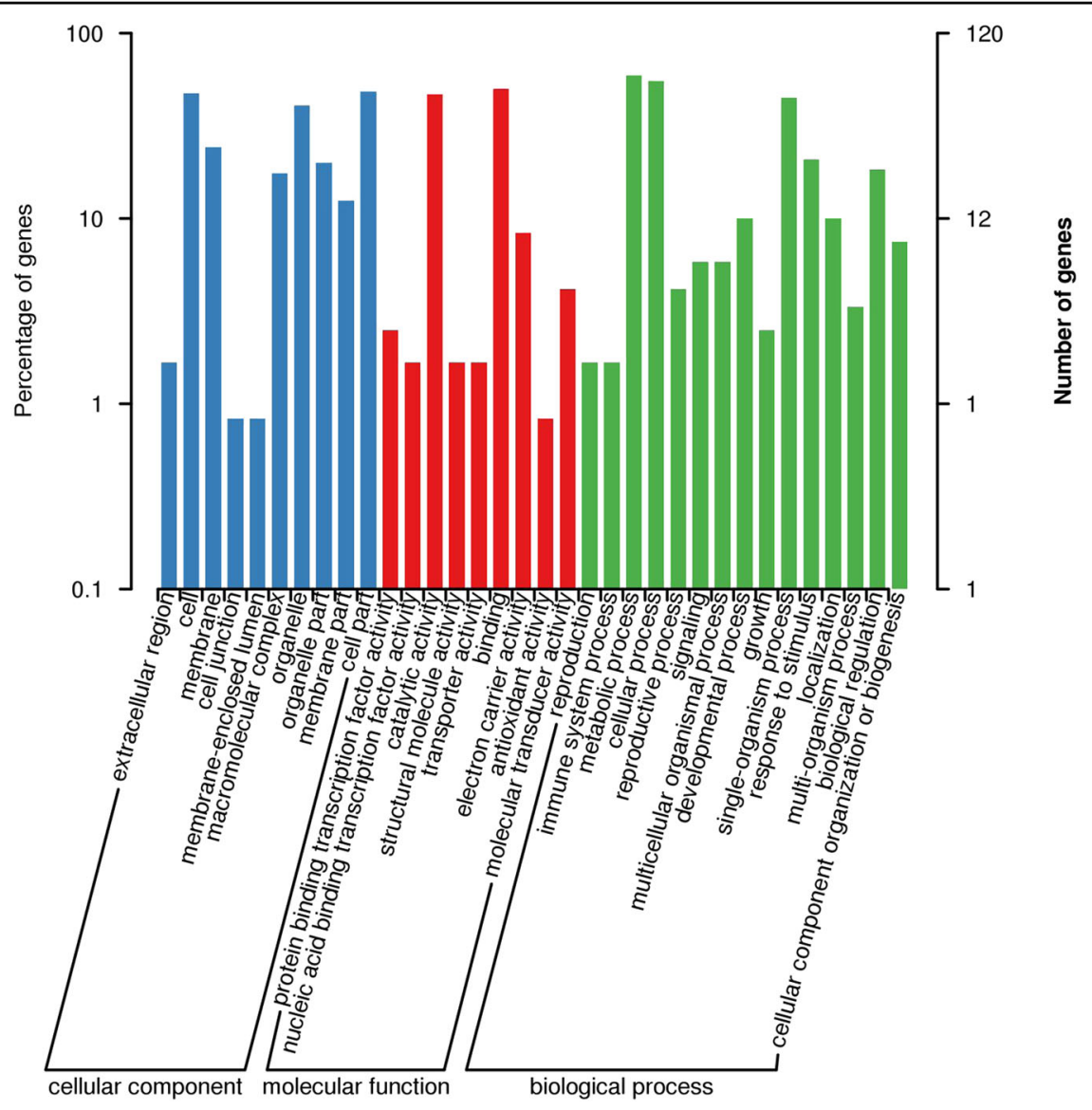

Fig. 6 Numbers of willow unigenes in each functional category in the confined IESL in S. triandra

Table 5 Classification and function of important genes in the confined IESL in chromosome XV of S. triandra

\begin{tabular}{lllll}
\hline Gene ID & Start & End & Strand & Function distribution annotation \\
\hline EVM0002598 & $12,380,227$ & $12,385,512$ & - & Microtubule motor activity \\
EVM0006532 & $13,615,059$ & $13,624,340$ & - & Microtubule motor activity \\
EVM0048283 & $13,638,084$ & $13,638,395$ & - & Microtubule motor activity \\
Salix_newGene_14 & $14,399,846$ & $14,402,709$ & + & Microtubule motor activity \\
Salix_newGene_6 & $13,351,098$ & $13,354,415$ & + & Microtubule motor activity \\
Salix_newGene_7 & $13,354,476$ & $13,357,328$ & + & Microtubule motor activity \\
EVM0038350 & $12,867,149$ & $12,869,132$ & + & Methyltransferase activity \\
EVM0005130 & $13,655,785$ & $13,658,118$ & - & Myb-like DNA-binding domain \\
Salix_newGene_28 & $14,013,632$ & $14,016,788$ & - & RNA-directed RNA polymerase activity \\
EVM0008632 & $13,406,181$ & $13,414,288$ & - & RNA processing and modification \\
EVM0045351 & $11,991,112$ & $12,004,061$ & + & Mitogen-activated protein kinase (MAP kinase) domain \\
Salix_newGene_2 & $12,380,227$ & $12,385,512$ & - & LRR receptor-like serine/threonine-protein kinase \\
\hline
\end{tabular}




\section{Acknowledgements}

This work was funded by the National Key Research and Development Plan of China (2016YFD0600101), the National Natural Science Foundation (31800562), and the Youth Elite Science Sponsorship Program of CAST (YESS). This work was also supported by the Priority Academic Program Development Program of Jiangsu Province.

\section{Author contributions}

W.L., H.W. and Y.C. participated in all the experiments and data analyses and drafted the manuscript. X.L. established the mapping pedigree and conducted phenotyping. Y.C. and T.Y. finalized the manuscript

\section{Conflict of interest}

The authors declare that they have no conflict of interest.

Supplementary Information accompanies this paper at (https://doi.org/ 10.1038/s41438-020-0289-1).

Received: 27 September 2019 Revised: 27 February 2020 Accepted: 2 March 2020

Published online: 01 May 2020

\section{References}

1. Darwin, C. R. (eds) The different forms of flowers on plants of the same species (John Murray, London, 1877).

2. Renner, S. S. The relative and absolute frequencies of angiosperm sexual systems: dioecy, monoecy, gynodioecy, and an updated online database. Am. J. Bot. 101, 1588-1596 (2014)

3. Vyskot, B. \& Hobza, R. The genomics of plant sex chromosomes. Plant Sci. 236, 126-135 (2015)

4. Otto, S. P. et al. About PAR: the distinct evolutionary dynamics of the pseudoautosomal region. Trends Genet. 27, 358-367 (2011).

5. Charlesworth, D. Plant sex chromosomes. Annu. Rev. Plant Biol. 67, 397-420 (2016).

6. Harkess, A. \& Leebens-Mack, J. A century of sex determination in flowering plants. J. Hered. 108, 69-77 (2017).

7. Dai, $X$. et al. The willow genome and divergent evolution from poplar after the common genome duplication. Cell Res. 24, 1274-1277 (2014).

8. Tuskan, G. A. et al. The genome of black cottonwood, Populus trichocarpa (Torr. \& Gray). Science 313, 1596-1604 (2006).

9. Peto, H. F. Cytology of poplar species and natural hybrids. Can. J. Res. 16 445-455 (1938).

10. Van Buijtenen, J. \& Einspahr, D. Note on the presence of sex chromosomes in Populus tremuloides. Bot. Gaz. 121, 60-61 (1959).

11. Almeida, P. et al. Single-molecule genome assembly of the Basket Willow, Salix viminalis, reveals earliest stages of sex chromosome expansion. Preprint at https://www.biorxiv.org/content/10.1101/589804v1.full (2019).

12. Yin, $\mathrm{T}$. et al. Genome structure and emerging evidence of an incipient sex chromosome in Populus. Genome Res. 18, 422-430 (2008).

13. Gaudet, M. et al. Genetic linkage maps of Populus nigra L. including AFLPS, SSRs, SNPs, and sex trait. Tree Genet. Genomes 4, 25-36 (2008).

14. Pakull, B., Groppe, K., Meyer, M., Markussen, T. \& Fladung, M. Genetic linkage mapping in aspen (Populus tremula L. and Populus tremuloides Michx.). Tree Genet. Genomes 5, 505-515 (2009).

15. Paolucci, I. et al. Genetic linkage maps of Populus alba L and comparative mapping analysis of sex determination across Populus species. Tree Genet. Genomes 6, 863-875 (2010).

16. Kersten, B., Pakull, B. \& Fladung, M. Genomics of sex determination in dioecious trees and woody plants. Trees 31, 1113-1125 (2017).

17. Pakull, B., Kersten, B., Lüneburg, J. \& Fladung, M. A simple PCR-based marker to determine sex in aspen. Plant Biol. 17, 256-261 (2015).

18. Caseys, C., Stölting, K. N., Barbará, T., González-Martínez, S. C. \& Lexer, C. Patterns of genetic diversity and differentiation in resistance gene clusters of two hybridizing European Populus species. Tree Genet. Genomes 11, 81 (2015).

19. Tuskan, G. A et al. The obscure events contributing to the evolution of an incipient sex chromosome in Populus: a retrospective working hypothesis. Tree Genet Genomes 8, 559-571 (2012).
20. Bräutigam, K. et al. Sexual epigenetics: gender-specific methylation of a gene in the sex determining region of Populus balsamifera. Sci. Rep. 7, 45388 (2017).

21. Chen, Y., Wang, T., Fang, L., Li, X. \& Yin, T. Confirmation of single-locus sex determination and female heterogamety in willow based on linkage analysis. PLOS ONE 11, e0147671 (2016)

22. Hou, J. et al. Different autosomes evolved into sex chromosomes in the sister genera of Salix and Populus. Sci. Rep. 5, 9076 (2015).

23. Pucholt, P., Rönnberg-Wästljung, A. C. \& Berlin, S. Single locus sex determination and female heterogamety in the basket willow (Salix viminalis L.). Heredity 114, 575 (2015).

24. Pucholt, P., Hallingbäck, H. R. \& Berlin, S. Allelic incompatibility can explain female biased sex ratios in dioecious plants. BMC Genomics 18, 251 (2017).

25. Pucholt, P., Wright, A. E., Conze, L. L., Mank, J. E. \& Berlin, S. Recent sex chromosome divergence despite ancient dioecy in the willow Salix viminalis. Mol. Biol. Evol. 34, 1991-2001 (2017).

26. Zhou, R. et al. A willow sex chromosome reveals convergent evolution of complex palindromic repeats. Genome Biol. 21, 1-19 (2020).

27. Jalas, J. \& Suominen, J. (eds) Atlas florae europaea: distribution of vascular plants in Europe (Cambridge University Press, 1988).

28. Hjältén, J. et al. Variable responses of natural enemies to Salix triandra phenotypes with different secondary chemistry. Oikos 116, 751-758 (2007).

29. Sannikova, E., Popova, O. \& Frolova, O. Determination of flavonoids of willow triandra (Salix triandra L.), growing in the north Caucasus. Pharm. Pharmacol. 4 56-67 (2016).

30. Baird, N. A. et al. Rapid SNP discovery and genetic mapping using sequenced RAD markers. PLoS ONE 3, e3376 (2008).

31. Li, H. \& Durbin, R. Fast and accurate short read alignment with Burrows-Wheeler transform. Bioinformatics 25, 1754-1760 (2009).

32. McKenna, A. et al. The genome analysis toolkit: a MapReduce framework for analyzing next-generation DNA sequencing data. Genome Res. 20, 1297-1303 (2010).

33. Depristo, M. A., Banks, E., Poplin, R., Garimella, K. V. \& Daly, M. J. A framework for variation discovery and genotyping using next-generation DNA sequencing data. Nat. Genet. 43, 491-498 (2011).

34. Kosambi, D. D. The estimation of map distance from recombination values. Ann. Eugen. 12, 172-175 (1943).

35. Liu, H., Cao, F., Yin, T. \& Chen, Y. A highly dense genetic map for Ginkgo biloba constructed using sequence-based markers. Front. Plant Sci. 8 1041 (2017).

36. West, M. A. L. High-density haplotyping with microarray-based expression and single feature polymorphism markers in Arabidopsis. Genome Res. 16, 787 (2006).

37. Angela et al. Genetic improvement of willow for bioenergy and biofuels. J. Integr. Plant Biol. 53, 151-165 (2011).

38. Dickmann, D. I., Kuzovkina, J., Isebrands, J. G. \& Richardson, J. Poplars and willows of the world, with emphasis on silviculturally important species. Poplars Willows Trees Soc. Environ. 22, 8 (2014).

39. Slavov, G. T. \& Zhelev, P. In Genetics and Genomics of Populus. Plant Genetics and Genomics: Crops and Models (eds. Jansson, S., Bhalerao, R., \& Groover, A.) Vol. 8 (Springer, New York, 2010).

40. Mock, K. E. et al. Widespread triploidy in western North American aspen (Populus tremuloides). PLOS ONE 7, e48406 (2012).

41. Serapiglia, M. J. et al. Ploidy level affects important biomass traits of novel shrub willow (Salix) hybrids. BioEnerg. Res. 8, 259-269 (2015).

42. Ashman, T.-L., Kwok, A. \& Husband, B. Revisiting the dioecy-polyploidy association: alternate pathways and research opportunities. Cytogenet. Genome Res. 140, 241-255 (2013)

43. Kersten, B., Pakull, B., Groppe, K., Lueneburg, J. \& Fladung, M. The sex-linked region in $P$ opulus tremuloides Turesson 141 corresponds to a pericentromeric region of about two million base pairs on $P$. trichocarpa chromosome 19. Plant Biol. 16, 411-418 (2014).

44. Geraldes, A. et al. Recent $Y$ chromosome divergence despite ancient origin of dioecy in poplars (Populus). Mol. Ecol. 24, 3243-3256 (2015)

45. Temmel, N. A., Rai, H. S. \& Cronk, Q. C. Sequence characterization of the putatively sex-linked Ssu72-like locus in willow and its homologue in poplar. Botany 85, 1092-1097 (2007)

46. Ming, R., Bendahmane, A. \& Renner, S. S. Sex chromosomes in land plants. Ann. Rev. Plant Biol. 62, 485-514 (2011).

47. Pilkington, S. et al. Genetic and cytological analyses reveal the recombination landscape of a partially differentiated plant sex chromosome in kiwifruit. BMC Plant Biol. 19, 172 (2019) 
48. Wang, J. et al. Sequencing papaya $X$ and $Y^{h}$ chromosomes reveals molecular basis of incipient sex chromosome evolution. Proc. Natl Acad. Sci. 109, 13710-13715 (2012)

49. Seefelder, S., Ehrmaier, H., Schweizer, G. \& Seigner, E. Male and female genetic linkage map of hops, Humulus lupulus. Plant Breed. 119, 249-255 (2000).

50. Lengerova, M., Moore, R. C., Grant, S. R. \& Vyskot, B. The sex chromosomes of Silene latifolia revisited and revised. Genetics 165, 935-938 (2003).

51. Sun, Y., Svedberg, J., Hiltunen, M., Corcoran, P. \& Johannesson, H. Large-scale suppression of recombination predates genomic rearrangements in Neurospora tetrasperma. Nat. Commun. 8, 1140 (2017).

52. Mariotti, B., Manzano, S., Kejnovský, E., Vyskot, B. \& Jamilena, M. Accumulation of Y-specific satellite DNAs during the evolution of Rumex acetosa sex chromosomes. Mol. Genet. Genomics 281, 249 (2009).

53. Zhang, W., Wang, X., Yu, Q., Ming, R. \& Jiang, J. DNA methylation and heterochromatinization in the male-specific region of the primitive $Y$ chromosome of papaya. Genome Res. 18, 1938-1943 (2008).

54. Jamilena, M., Mariotti, B. \& Manzano, S. Plant sex chromosomes: molecular structure and function. Cytogenet. Genome Res. 120, 255-264 (2008).
55. Zhou, R. et al. Characterization of a large sex determination region in Salix purpurea L.(Salicaceae). Mol. Genet. Genomics 293, 1437-1452 (2018).

56. Ainsworth, C. Boys and girls come out to play: the molecular biology of dioecious plants. Ann. Bot. 86, 211-221 (2000).

57. Diggle, P. K. et al. Multiple developmental processes underlie sex differentiation in angiosperms. Trends Genet. 27, 368-376 (2011).

58. Akagi, T. et al. A Y-encoded suppressor of feminization arose via lineagespecific duplication of a cytokinin response regulator in kiwifruit. Plant Cell 30, 780-795 (2018).

59. Akagi, T., Henry, I. M., Tao, R. \& Comai, L. A Y-chromosome-encoded small RNA acts as a sex determinant in persimmons. Science 346, 646-650 (2014).

60. Cai, G. \& Cresti, M. Organelle motility in the pollen tube: a tale of 20 years. J. Exp. Bot. 60, 495-508 (2008).

61. Zhu, Q. H., Ramm, K., Shivakkumar, R., Dennis, E. S. \& Upadhyaya, N. M. The ANTHER INDEHISCENCE1 gene encoding a single MYB domain protein is involved in anther development in rice. Plant Physiol. 135, 1514-1525 (2004).

62. Ichimura, K. et al. Mitogen-activated protein kinase cascades in plants: a new nomenclature. Trends Plant Sci. 7, 301-308 (2002). 